\title{
ADJUSTMENT OF CODE RANGING OF GNSS OBSERVATIONS
}

Gauss-Jacobi combinatorial algorithm is a very useful method to adjust code ranging of global navigation satellite observations to find the systematic errors caused by radio waves passing through the atmosphere. The method is used as alternative adjustment technique to the least square method. While, the least square adjustment requires the linearization of nonlinear functions, combinatorial approach seems to be simplier in direct processing the nonlinear models. The diagnostics of observation errors consists in comparison of an evaluated partial positional norm determined in the partial mathematical models with the global one determine by robust estimation.

Keywords: Code ranging, combinatorial algorithm, observation errors, positional norm.

\section{Introduction}

Global navigation satellite system (GNSS) involves two observation techniques to measure the range (pseudorange) between satellite and receiver. Code pseudorange measurement, in short code ranging, is used to measure the time difference between the received code and generated replica code. Phase pseudorange measurement, phase ranging, is based on measurement of phase difference between the received and generated signal. The received carrier is Doppler shifted due to the mutual motion of satellite and receiver [1]. The accuracy of pseudorange obtained by the code or phase ranging depends on random and systematic influences. The whole system error involves the particular errors of determination of satellite time and position, signal transition errors caused by using the incorrect atmospheric model comprising the influence of ionospheric and stratospheric refraction and topography errors known as multipath effect. The great part of system error is generated by a receiver failure, which comprises a variation of phase centre and clock errors. The paper is devoted to a numerical analysis of code ranging especially to diagnose influence of systematic errors by applying the GaussJacobi algorithm [2, 3 and 4].

\section{Numerical Approaches of Code Ranging}

Gauss-Jacobi algorithm is often used to estimate unknown parameters in nonlinear models [5 and 6]. In geodesy, it is applied as an alternative method to nonlinear Gauss-Markov model, which uses Taylor series for linearization. Application of GaussJacobi combinatorial algorithm in diagnostics of systematic errors in code ranging assumes to arrange the vector of receiver position, which involves the differences between system time $t$ and time delay $\tau_{i}$ of a signal which originates from a satellite position as follows [7, 8 and 9]:

$\mathbf{r}^{T}\left(t-\tau_{i}\right)=\left(r_{1}\left(t-\tau_{1}\right), r_{2}\left(t-\tau_{2}\right), \ldots, r_{n}\left(t-\tau_{n}\right)\right)$

Vector of measurements, which is represented by the pseudorange values $d_{i}(t)$ and observed in time $t$ can be defined by the formula:

$\mathbf{y}^{T}=\left(d 1(t), d_{2}(t), \ldots, d_{n}(t)\right)$

Providing $\Delta t_{u}(t)$ is correction of the system time of a receiver, $\Delta t_{i}\left(t-\tau_{i}\right)$ is correction of the system time of a satellite and $c$ is signal career, the basic formula for code ranging is as follows:

$d(t)=c \tau_{i}+c \cdot \Delta t_{u}(t)-c \Delta t_{i}\left(t-\tau_{i}\right)$,

where $c \tau_{i}=D(t)$ represents the real range, $c \Delta t_{u}(t)=b(t)$ is the unknown parameter because the system time varies in each satellite and correction of system time of a receiver is an unknown value. The last quantity of an equation (3) $c \cdot \Delta_{i}\left(t-\tau_{i}\right)=b\left(t-\tau_{i}\right)$ is corrected range, which involves

\footnotetext{
* ${ }^{1} \mathrm{Jana}$ Izvoltova, ${ }^{1}$ Peter Pisca, ${ }^{2}$ Pavel Cernota, ${ }^{3}$ Marian Mancovic

${ }^{1}$ Department of Geodesy, Faculty of Civil Engineering, University of Zilina, Slovakia

${ }^{2}$ Institute of Geodesy and Mining, Technical University of Ostrava, Czech Republic

${ }^{3}$ Institute of Continuing Education, University of Zilina, Slovakia

E-mail: jana.izvoltova@fstav.uniza.sk
} 
the time correction of satellites acquired from almanac. Then, the function of code pseudorange can be rewritten into a common formula:

$$
\bar{d}(t)=d(t)+b\left(t-\tau_{i}\right)=D(t)+b(t) .
$$

\section{Gauss-Jacobi Combinatorial Algorithm}

In conformity with the previously described observation model (4), the mathematical model of code ranging is demonstrated according to formulas [3 and 4] as follows:

$$
\begin{aligned}
& \mathbf{y}=\sqrt{\left(\mathbf{r}(t)-\mathbf{r}\left(t-\tau_{i}\right)\right)^{T}\left(\mathbf{r}(t)-\mathbf{r}\left(t-\tau_{i}\right)\right)}+ \\
& +\mathbf{b}(t)+\mathbf{e}
\end{aligned}
$$

where the vector of receiver position $\mathbf{r}(t)$, vector of clock corrections $\mathbf{b}(t)$ and vector of residuals e belong to the unknown parameters of model, which are estimated by Gauss-Jacobi combinatorial algorithm. It is a very useful method to ensure both procedures, to estimate the appropriate pseudorange and to identify the possible systematic influence on mathematical model (5). The principle of this method is in creating the minimal number of partial combinations of the model, which satisfies the combinatorial number:

$$
C_{k}=\left(\begin{array}{l}
n \\
u
\end{array}\right)=\frac{n !}{u !(n-u) !} .
$$

The rank of the particular matrices of the $p$-models depends on the number of unknown parameters $u$. The solution of the combinatorial algorithm consists in estimating the introductory positional parameters $\beta_{i}^{p}$ from the $i$-th equation of $p$-model as follows

$\beta_{i}^{p}=\left(\begin{array}{c}\beta_{1}^{p} \\ \beta_{2}^{p} \\ \vdots \\ \beta_{u}^{p}\end{array}\right)=\mathbf{A}_{i}^{p^{-1}} \mathbf{y}_{i}^{p}$

and in defining the partial matrices:

$$
\mathbf{G}_{i}^{p}=\mathbf{A}_{i}^{p^{T}} \mathbf{P}_{i}^{p} \mathbf{A}_{i}^{p},
$$

which have to be positive definite and regular because of their inversion. The unknown parameters of the mathematical model (5) are represented by the weighted averages which are estimated from the equation:

$\bar{\beta}=\left(\begin{array}{c}\bar{\beta}_{1} \\ \bar{\beta}_{2} \\ \vdots \\ \bar{\beta}_{u}\end{array}\right)=\left(\mathrm{G}_{i}^{p}+\mathrm{G}_{i+1}^{p}\right)^{-1}\left(\mathrm{G}_{i}^{p} \mid \mathrm{G}_{i+1}^{p}\right)\left(\frac{\beta_{i}^{p}}{\boldsymbol{\beta}_{i+1}^{p}}\right)$
The proper identification of the systematic influence in the model consists in comparison of a partial combinatorial positional norm estimated from the $i$-th model:

$K_{i}^{p}=\sqrt{x_{i}^{2}+y_{i}^{2}+z_{i}^{2}}$

with the median positional norm $K_{\text {med }}$ in the case of robust estimation or with the "global" positional norm $K_{\bar{\beta}}$ estimated average from the whole model.

\section{Verification of Efficiency of Combinatorial Algorithm}

The diagnostics of systematic effect on final pseudorange belongs to the fundamental approaches of each GNSS developer and, therefore, there are a lot of hardware and software solutions in engineering practice. Differential GNSS seems to be one of the best methods to avoid this effect by using it in the process of determining the precise receiver position in geodesy applications [1]. However, the actual ionospheric and tropospheric model, precise time delay and satellite ephemerids are always important to know. The efficiency of the Gauss-Jacobi combinatorial algorithm was verified on real raw data arranged in "rinex" format, which were obtained by the static GNSS method. Inter alia, the "rinex" data file contains the number of visible satellites $(\mathrm{G} 4, \mathrm{G} 31, \mathrm{G} 29, \ldots)$ and the appropriate carrier phases (L1, L2), pseudo ranges (P1, P2) and Doppler frequency, as can be seen in Fig. 1.

The precise satellite's position needed for pseudorange calculations is defined by Cartesian coordinates in Global Reference Frame IGS08 and is accessible as a product of NASA and ESA in "sp3" format. For $n=13$ satellites and $u=4$ unknown parameters $(x, y \mathrm{z}$, bias $)$, we calculated the minimal number of satellite combinations $C_{k, \min }=715$.The unknown parameters of the partial models were estimated according to the equation (7) to calculate the partial positional norms according to the equation (10):

$K_{i}^{p}=\sqrt{\mathbf{r}_{i}(t-\tau)^{T} \mathbf{r}_{i}(t-\tau)}$.

The significant difference between the positional norm in model average

$K_{\bar{\beta}}=\sqrt{\mathbf{r}_{\beta}(t-\tau)^{T} \mathbf{r}_{\beta}(t-\tau)}$

and the positional norm in median:

$K_{\text {med }}=\sqrt{\mathbf{r}_{\text {med }}(t-\tau)^{T} \mathbf{r}_{\text {med }}(t-\tau)}$ 


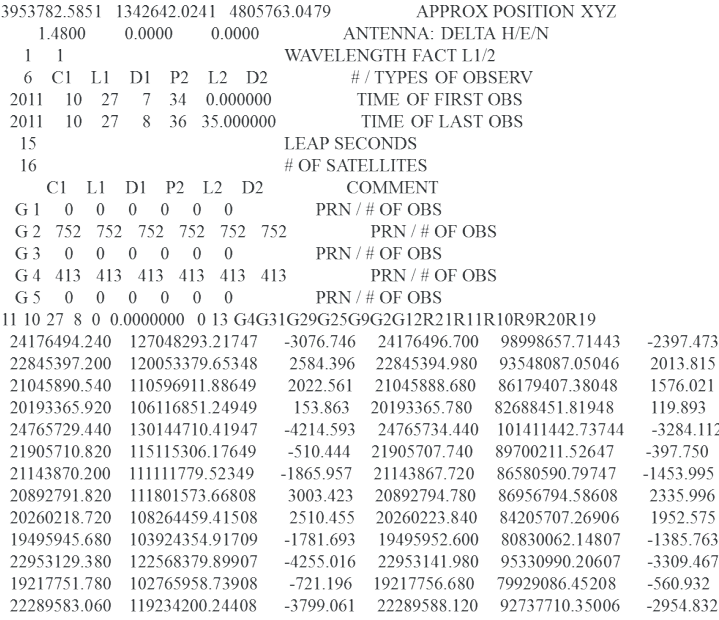

Fig. 1 Demonstration of raw GNSS data in "rinex" format

gives the view of error influence on observation data. The receiver position calculated from the code ranging obtained from satellites R11 and R19 seems to be influenced by observation error, as we can see from the positional norm differences displayed in Table 1. In practice, the differences from the positional norm demonstrate the pseudorange error, which can be caused by the multipath influence or improper satellite geometry. While, the first reason is easy to eliminate by an appropriate software, the second one is registered as PDOD value in a receiver.

Differences between partial and global positional norms Table 1

\begin{tabular}{|c|c|c|c|c|}
\hline \multirow{2}{*}{$\begin{array}{c}\text { Combination } \\
\text { number }\end{array}$} & \multirow{2}{*}{$\begin{array}{c}\text { Partial combinations } \\
\text { of satellites }\end{array}$} & \multirow{2}{*}{$\begin{array}{l}\text { Positional } \\
\text { Norm in km }\end{array}$} & \multicolumn{2}{|c|}{ Deviations } \\
\hline & & & Average norm & Median norm \\
\hline 1 & G4 G31 G29 G25 & 6366.334 & -0.019 & -0.013 \\
\hline 2 & G4 G29 G25 G9 & 6366.320 & -0.032 & -0.026 \\
\hline 3 & $\mathrm{G} 4 \mathrm{G} 25 \mathrm{G} 9 \mathrm{G} 2$ & 6366.302 & -0.050 & -0.044 \\
\hline 4 & G4 G9 G2 G12 & 6366.328 & -0.024 & -0.019 \\
\hline 5 & G4 G2 G12 R21 & 6366.371 & 0.019 & 0.025 \\
\hline 6 & G4 G12 R21 R11 & 6366.347 & -0.005 & 0.000 \\
\hline 7 & G4 R21 R11 R10 & 6366.352 & 0.000 & 0.006 \\
\hline 8 & G4 R11 R10 R19 & 6366.589 & 0.237 & 0.243 \\
\hline 9 & G4 R10 R19 R20 & 6366.181 & -0.171 & -0.166 \\
\hline 10 & G4 R19 R20 R9 & 6366.383 & 0.031 & 0.036 \\
\hline 11 & G2 G4 G31 G29 & 6366.345 & -0.007 & -0.002 \\
\hline 12 & G2 G31 G29 G25 & 6366.336 & -0.016 & -0.010 \\
\hline 13 & $\mathrm{G} 2 \mathrm{G} 29 \mathrm{G} 25 \mathrm{G} 9$ & 6366.335 & -0.016 & -0.011 \\
\hline 14 & G2 G25 G9 G12 & 6366.376 & 0.024 & 0.029 \\
\hline 15 & G2 G9 G12 R21 & 6366.346 & -0.006 & 0.000 \\
\hline 16 & G2 R12 R21 R11 & 6366.351 & 0.000 & 0.005 \\
\hline 17 & G2 R21 R11 R10 & 6366.138 & -0.214 & -0.209 \\
\hline 18 & G2 R11 R10 R19 & 6365.988 & -0.363 & -0.358 \\
\hline 19 & G2 R10 R19 R20 & 6366.358 & 0.006 & 0.012 \\
\hline 20 & G2 R19 R20 R9 & 6366.397 & 0.045 & 0.050 \\
\hline 21 & G31 G29 G25 G9 & 6366.352 & 0.000 & 0.005 \\
\hline 22 & G31 G25 G9 G2 & 6366.342 & -0.010 & -0.004 \\
\hline 23 & G31 G9 G2 G12 & 6366.346 & -0.006 & -0.001 \\
\hline 24 & G31 G2 G12 R21 & 6366.346 & -0.005 & 0.000 \\
\hline 25 & G31 G12 R21 R11 & 6366.346 & -0.006 & 0.000 \\
\hline
\end{tabular}

The differences of the partial norms from the global positional norm in median can also be illustrated in Fig. 2.

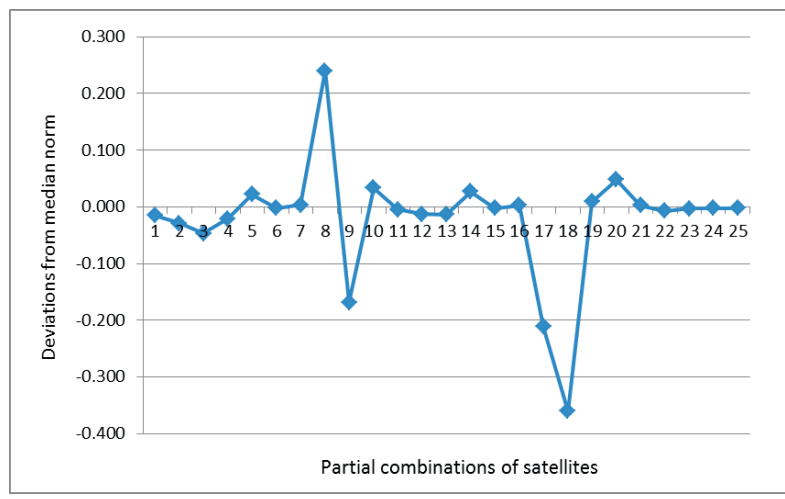

Fig. 2 Comparison of partial positional norm with the global one in median

\section{Conclusion}

The primary purpose of using Gauss-Jacobi combinatorial algorithm was to amend the endless iterative methods of parameter estimation with the creation of the minimal number of mathematical combinations of observed data. The practical application of this method demonstrates its strength to estimate unknown parameters in nonlinear mathematical models with current diagnostics of observation errors with systematic effect. Comparing the global positional norm with a partial one estimated in $i$-th partial model refers to code ranging error existence probably caused by multipath effect. Gauss-Jacobi combinatorial algorithm enables us to identify improper satellite position in a given system time.

\section{Acknowledgement}

This article is a result of the implementation of the project VEGA No. 1/0597/14 "Analysis of methods used to measure the unconventional railway track construction from the point of view of accuracy and reliability" supported by the Scientific Grant Agency of the Ministry of Education, Science, Research and Sport of the Slovak Republic and the Slovak Academy of Sciences. 


\section{COMMNICOIIIONS}

\section{References}

[1] RAO, B., R., K., SARMA, A., D., KUMAR, Y., R.: Technique to Reduce Multipath GPS Signals. Research Communications, Current Science, vol. 90, No. 2, 2006, India.

[2] GRAFAREND, E. W.: Linear and Nonlinear Models. Walter de Gruyter Berlin : New York, 2006, p. 752.

[3] HEFTY, J., HUSAR, L.: Satellite Geodesy, STU : Bratislava, 2003, 186 p.

[4] HOFMANN WELENHOF, B., MORITZ, H.: Physical Geodesy, $2^{\text {nd }}$ ed., Springer Wien : New York, 2006, ISBN-13978-3-211-33544-4, $403 \mathrm{p}$.

[5] AWANGE, J. L., GRAFAREND, E. W.: Solving Algebraic Computational Problems in Geodesy and Geoinformatics, Springer Berlin : New York, 2005

[6] GASINCOVA, S., GASINEC, J.: Adjustment of Positional Geodetic Networks by Unconventional Estimations, Acta Montanistica Slovaca, 15/1, 2010, ISSN 1335-1788, pp. 71-85.

[7] HRDINA, Z., PANEK, P., VEJRAZKA, F.: Position Determination by Radio Ranging. CVUT : Praha, 1999.

[8] KOCH, K., R.: Parameter Estimation and Hypothesis Testing in Linear Models. Springer-Verlag : Berlin, 1988, p. 378.

[9] MUZIK, J., KOVARIK, K., SITANYOVA, D.: Meshless Analysis of an Embankment Using Local Galerkin Radial Point Interpolation Method, Communications - Scientific Letters of University of Zilina, 2/2013, ISSN 1335-4205, pp. 34-40. 